\title{
EVALUATION OF EQUIVALENT VISION TECHNOLOGIES FOR SUPERSONIC AIRCRAFT OPERATIONS
}

\author{
Lynda J. Kramer, Steven P. Williams, Susan J. Wilz, Jarvis (Trey) J. Arthur III, and Randall E. Bailey \\ NASA Langley Research Center, Hampton, VA
}

\begin{abstract}
Twenty-four air transport-rated pilots participated as subjects in a fixed-based simulation experiment to evaluate the use of Synthetic/Enhanced Vision (S/EV) and eXternal Vision System (XVS) technologies as enabling technologies for future all-weather operations. Three head-up flight display concepts were evaluated a monochromatic, collimated Head-up Display (HUD) and a color, non-collimated XVS display with a fieldof-view (FOV) equal to and also, one significantly larger than the collimated HUD. Approach, landing, departure, and surface operations were conducted. Additionally, the apparent angle-of-attack (AOA) was varied (high/low) to investigate the vertical field-ofview display requirements and peripheral, side window visibility was experimentally varied. The data showed that lateral approach tracking performance and lateral landing position were excellent regardless of the display type and AOA condition being evaluated or whether or not there were peripheral cues in the side windows. Longitudinal touchdown and glideslope tracking were affected by the display concepts. Larger FOV display concepts showed improved longitudinal touchdown control, superior glideslope tracking, significant situation awareness improvements and workload reductions compared to smaller FOV display concepts.
\end{abstract}

\section{Introduction}

NASA is conducting research into technologies for reducing the impact of aircraft sonic boom on people and the environment. A successful low-boom design drives the shaping and configuration of the vehicle. One such conceptual configuration is shown in Figure 1. As evident in this figure, pilot forward visibility is severely compromised as a result of the vehicle shaping.

Under the Fundamental Aeronautics (FA) Program, Supersonics project, NASA is performing fundamental research, development, test and evaluation of flight deck and related technologies to support these low-boom, supersonic configurations by use of an eXternal Vision System (XVS). XVS is a combination of sensor and display technologies intended to provide an equivalent level of safety and performance to that provided by forward-facing windows in today's aircraft.

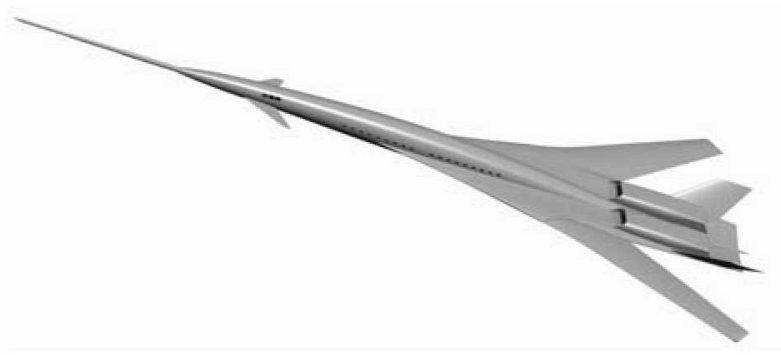

Figure 1. Conceptual Low-Boom Supersonic Aircraft Configuration.

Without XVS, the economic viability of a lowboom supersonic aircraft is questionable, since the lack of forward visibility by the pilot would severely restrict aircraft operations and airspace usage especially when the weather is clear and visibility conditions are unrestricted - i.e., when Visual Flight Rules (VFR) conditions prevail. Without XVS, a low-boom supersonic aircraft cannot "see-and-avoid."

Significant research was conducted under NASA's High Speed Research program during the 1990 s on the design and development issues associated with an XVS for a conceptual high-speed civil transport aircraft [1,2]. What emerged from this research (and still holds true today) is that the key challenge for an XVS design exists during VFR operations when it is assumed that the flight crew has natural visibility (even though they may be operating on an Instrument Flight Rules flight plan).

The driving XVS design standards emerged from the three tenets of VFR operations which are "see-andavoid", "see-and-follow", and "self-navigation". These VFR-type requirements are not unique to lowboom supersonic aircraft. For instance, an emerging challenge - being brought forth in the evolution of the National Airspace System to accommodate a 3-fold 
increase in air traffic - is the concept of Equivalent Visual Operations (EVO) [3]. The EVO concept is notionally the ability to achieve or even improve on the safety of current-day VFR operations, maintain the operational tempos of VFR, and even perhaps, retain VFR procedures - all independent of the actual weather and visibility conditions.

While EVO capability is not required for a successful supersonic aircraft, several of the technologies and operational requirements to develop EVO are in many ways equivalent to XVS. As such, current NASA research in the use of synthetic and enhanced vision systems (S/EVS) and other interface modalities as enabling technologies to help meet the EVO operational concept (which are being explored under the Aviation Safety Program, Integrated Intelligent Flight Deck project) are pertinent to this work [4-6]. A synthetic vision system (SVS) is a computer-generated image of the external scene topography, generated from aircraft attitude, highprecision navigation, and data of the terrain, obstacles, cultural features, and other required flight information that may be presented to a pilot on a Head-Up Display (HUD) or Primary Flight Display (PFD). An enhanced vision system (EVS) is an electronic means of providing a display (typically on a HUD) of the external scene by use of an imaging sensor, such as a Forward-Looking InfraRed (FLIR) or millimeter wave radar (MMWR). Both synthetic vision (SV) and enhanced vision (EV) are "vision-based" technologies intended to create, supplement, or enhance the natural vision of the pilot.

Paradoxically, EVO technologies strive to create VFR operations for today's aircraft when flying in actual Instrument Meteorological Conditions (IMC) whereas XVS technologies try to enable an IMC cockpit (i.e., low-boom supersonic aircraft) to operate under VFR. These two contrasting objectives share a need for research in the vision and visibility requirements to enable EVO or XVS. That is, what information and in what manner shall this information be delivered to the flight crew to conduct EVO, whether EVO is created on the "windowed" flight deck when flying in actual IMC or whether it is created because of intentional restrictions to the flight crew's external visibility due to aircraft structure and design.

An experiment was conducted to evaluate the feasibility of XVS/SVS/EVS to provide for all weather (visibility conditions) landing capability without the need (or ability) for a visual approach segment and to determine the interaction of XVS/EVS and peripheral vision cues for terminal area and surface operations. Another key element of the testing investigated the pilot's awareness and reaction to non-normal events (i.e., failure conditions) that were unexpectedly introduced into the experiment. These non-normals are critical determinants in the underlying safety of allweather operations.

This paper describes an experimental evaluation of field-of-view (FOV), collimation, and peripheral cues on pilot performance and subjective ratings of situation awareness and workload during terminal area operations. Further, the objective data from this test are being used to develop performance-based approach and landing standards which might establish a basis for future all-weather landing certification.

\section{Method}

\section{Subjects}

Twenty-four pilots, representing 6 airlines participated in the experiment. All participants had previous experience flying Head-Up Displays (HUDs). The subjects had an average of 1788 hours of HUD flying experience and an average of 20.4 years and 13.8 years of commercial and military flying experience, respectively.

\section{Simulation Facility}

The experiment was conducted in the Integration Flight Deck (IFD) simulation facility (Figure 2) at NASA Langley Research Center. The IFD emulates a Boeing B-757-200 aircraft and provides researchers with a full-mission simulator capability. The cab is populated with flight instrumentation and pilot controls, including the overhead subsystem panels, to replicate the B-757 aircraft. The collimated out-thewindow (OTW) scene is produced by an Evans and Sutherland Image Generator 4530 graphics system providing approximately 200 degrees horizontal by 40 degrees vertical FOV at 26 pixels per degree. The forward windows were masked for this experiment but the side windows were unblocked to test the effects of peripheral cues (with and without) during approach, landing, taxi, and departure operations. 


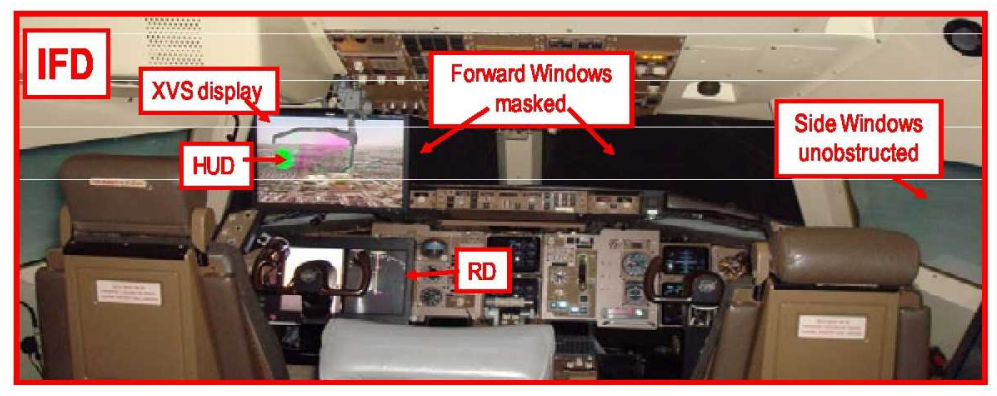

Figure 2. IFD Simulation Facility with HUD, XVS Display and Head-Down Research Display (RD).

The evaluation pilot (EP) occupied the left seat, as the Pilot Flying (PF) for this experiment. The left seat included an overhead HUD projection unit, a 22 inch diagonal liquid crystal display (LCD) (referred to as the XVS display) and a head-down research display (RD) (Figure 2). The right seat was occupied by a principal investigator (PI) who acted as First Officer during data collection. The PI aided the EP by providing callouts during taxi and performing airplane configuration tasks during departure runs.

\section{Head-Up Display}

The HUD was collimated and subtended approximately $26^{\circ}$ horizontal by $21^{\circ}$ vertical FOV. Note that to maintain conformality with the outside world, the FOV for the HUD imagery was fixed and could not be varied by the EP. The HUD presentation was written strictly in raster format from a video source (RS-343) input. The input consisted of a video mix of symbology and fused SV/EV imagery. The symbology included "haloing" to ensure that the symbology was highlighted against the scene imagery background. Fused SV/EV [5] imagery was chosen based on prior research that showed improvements in situation awareness and workload when using fused SV/EV imagery compared to when using imagery that was solely SV or EV. On initial approach to 600 feet height above terrain (HAT), the image was synthetic only and contained no EV imagery. Between 600 and $500 \mathrm{ft}$, the resulting imagery was a fusion between synthetic and enhanced vision, gradually transitioning to a complete FLIR image at 500 feet. From $500 \mathrm{ft}$ to touchdown, the image was FLIR only. Overall HUD brightness and contrast controls were provided to the pilot. In addition, the EP was able to independently adjust the flight symbology brightness relative to the raster imagery. The pilot also had a declutter control, implemented as a four-button castle switch on the left hand horn of the PF yoke. Four "declutter" states were available to the EP: 1) Symbology toggle (on/off); 2)
Imagery toggle (on/off); 3) All decluttered (no symbology or imagery); and 4) All displayed (both symbology and imagery).

\section{eXternal Vision System Display}

The XVS display subtended approximately $44^{\circ}$ horizontal by $34^{\circ}$ vertical FOV and was located approximately 19 inches from the pilot design eye point. The imagery on the XVS display was conformal with the OTW view just as the HUD imagery. However, this display differed from the HUD as it was larger, used color, and was not collimated. The XVS display emulated a camera view mounted on the outside of the aircraft with flight symbology overlaid on the scene. Thus, any items (e.g., traffic, approach lighting system, terrain, runway markings, etc.) that would be visible to a real camera system would be visible in the color camera imagery. This photorealistic camera imagery was unaffected by the outside weather to parametrically test for any interactions between display size and peripheral cues. The same declutter control described in the HUD section above was utilized with the XVS display.

\section{Head-Down Research Display}

A head-down RD was installed over the normal instruments on the left hand side of the IFD cockpit (Figure 2). The RD covered the captain's displays normal for a Boeing 757-200 with the exception of the analog standby instruments (attitude direction indicator, airspeed, and altitude). The RD used an 18.1-inch diagonal case containing two high brightness LCDs. The two separate LCD panels, each with XGA (1024x768) resolution, rendered a Size D (6.4 inch square viewable area) PFD and navigation display (ND).

\section{Symbology}

The same symbology set was used for the XVS and HUD concepts (Figure 3). The symbology 
included pathway guidance and a runway outline. The pathway symbology [7] ended at $500 \mathrm{ft}$ HAT and was replaced by a runway outline and a glideslope reference line. A runway outline symbol $(8000 \mathrm{ft} x$ $200 \mathrm{ft}$ ) was drawn using the threshold coordinates of the landing runway and the aircraft navigation solution to conformally position the symbol. A glideslope reference line was drawn at a descent angle of 3.1 degrees. Also, radar altitude was shown digitally underneath the flight path marker when below $500 \mathrm{ft}$ above ground level (AGL).

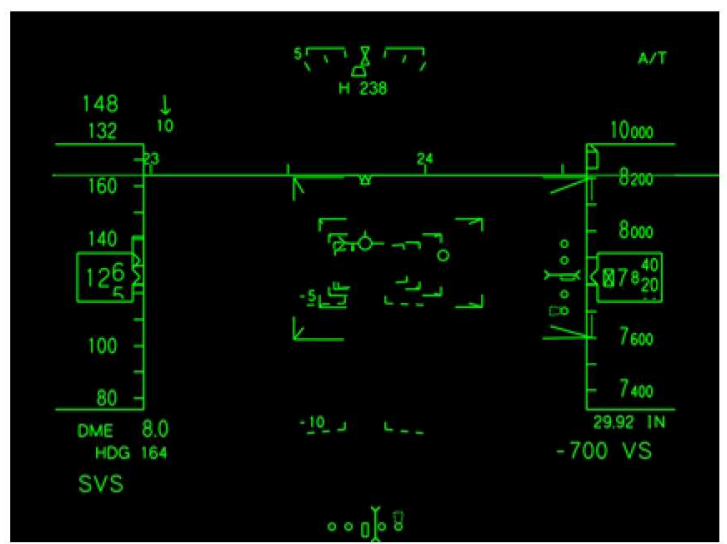

Figure 3. Head-Up Flight Display Symbology Format - Low AOA Condition Shown

A pitch-roll guidance cue ("ball") used modified pursuit guidance along the desired path [8]. Horizontal and vertical position of the ball reflects the track and flight path angles to fly to the center of the desired path. The path deviation indicators showed angular course deviation (i.e., glideslope and localizer-like) conditions by converting the linear path error data to angle errors and scaling in "dots." Glideslope and localizer raw data indicators which included a deviation scale and angular deviation indication were also provided (i.e., glideslope and localizer deviation).

\section{Independent Variable - Display Concepts}

Six head-up flight display concepts were evaluated by the EPs while flying approaches to Runway $16 \mathrm{R}$ at the Reno-Tahoe International Airport (airport identifier RNO). The head-down PFD and ND formats were invariant.

\section{Head-Up Flight Display Concepts}

The six head-up flight display concepts (Figure 4) were a $2 \times 3$ factorial combination of angle-of-attack, or AOA (low or high) and display type (HUD, XVS display with HUD FOV, or XVS display with full FOV).

The HUD installed in the RFD simulator uses a pitch bias value of 3 degrees. This means that the waterline (boresight) reference point of the HUD is 3 degrees above the center of the HUD. This bias tailors the placement of the HUD symbology for the B-757 simulator in its nominal operating flight conditions. In Figure 3, the HUD during this "low AOA" condition is shown. With the B757 at approximately 3 degrees in the approach conditions for this test, the flight path marker on short final is approximately at the center of the HUD.

One of the display design issues facing a supersonic aircraft is that they typically operate at high AOA conditions in the approach and landing, due to the high sweep-back wing angles and low camber typically desired for efficient supersonic cruise flight. For the flight deck designer, high AOA conditions on the approach drive the vertical FOV of windows to allow pilot visibility of the approach lights and touchdown zone (see FAA Advisory Circular AC25773-1 - Reference 9). For the Concorde, a droopednose was used to provide this visibility. By analogy, an XVS display, providing equivalent visibility, would require the same vertical FOV, particularly if conformal symbology and imagery create "electronic" visibility. As the AOA becomes very large, the recommended down-angle becomes significantly larger, following the so-called "3-second rule." Review of AC25-773-1 suggests that the substantiation for this requirement is vague and dated, particularly its relevance, as it might be applied to "electronic" visibility systems [9].

As an initial evaluation of this FOV down-angle requirement, a "high AOA" condition was simulated to compare to the "low AOA" condition. To minimize a confound in the experiment, actual high angle-of-attack conditions for the B757 were not simulated; otherwise, the flying qualities of the low AOA and high AOA conditions would be radically different. Instead, a pitch bias was introduced. 


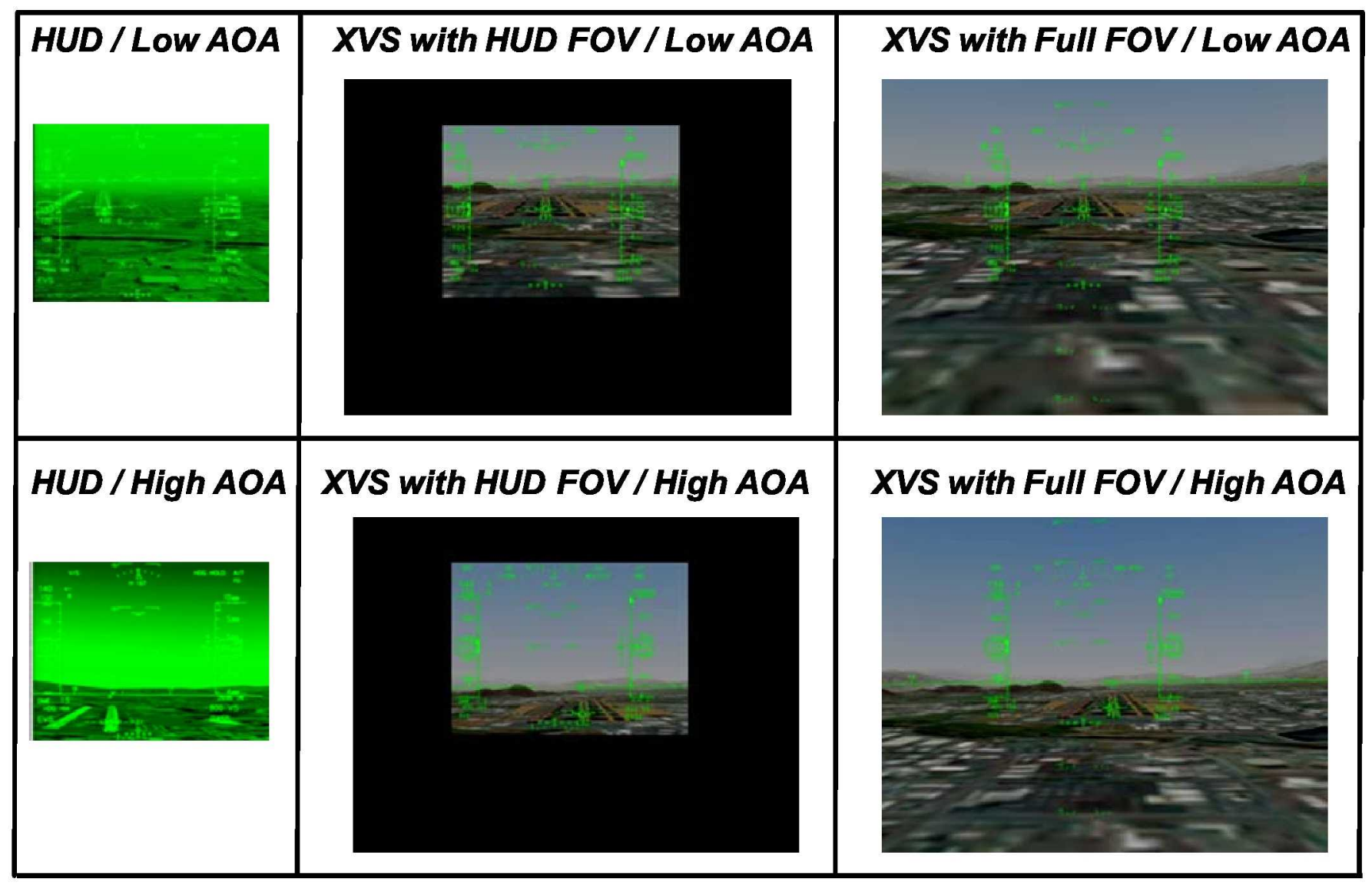

Figure 4. Head-Up Flight Display Concepts

The high AOA condition used an $8^{\circ}$ pitch bias to simulate an increased angle-of-attack to approximate that of a supersonic transport aircraft on approach (Figure 5), approximately an aircraft at $11^{\circ}$ angle-ofattack on approach. Comparing Figure 3 and Figure 5 shows the differences in HUD symbology. The pitch bias caused a "symbology cluster" with the flight path marker, guidance cue and localizer deviation scale/marker, especially with the smaller sized displays, while the pilots performed an approach on a 3.1 degree glide path to Runway $16 \mathrm{R}$.

The two AOA conditions were evaluated independently on either the monochromatic, collimated HUD or on the color, uncollimated XVS display. Two XVS display types were tested by simulating two different FOVs: 1) either the $26 \times 21$ degrees identical to the HUD FOV; or, 2) the full FOV of the XVS display (44 x 34 degrees). The symbology used in the XVS concepts was identical to that used in the HUD concept.
The XVS and HUD concepts were located in the same head-up positions so the aircraft boresight references for each display were co-located.

Simulated color camera imagery was mixed with the symbology and shown conformally on the color, uncollimated XVS display for both FOVs. The HUD was stowed to preclude blocking or distortion of the pilot's forward view when using the XVS display. The XVS display was turned off when the EP was evaluating a HUD concept. Note that the forward windows were masked for both display devices and the side windows were unobstructed.

\section{Head-Down Flight Display Concepts}

The PFD and ND closely resembled current transport aircraft equipage. However, guidance information was purposely removed from the PFD so that the EPs would focus on the head-up primary flight display concepts. The ND showed the RNO Runway 16R approach path, but it did not include any Enhanced Ground Proximity Warning System or 
Traffic Alert and Collision Avoidance System information.

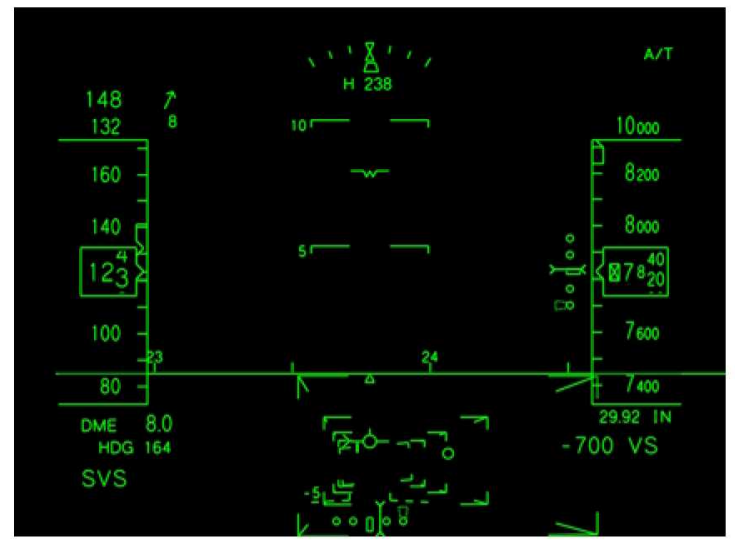

Figure 5. HUD When Flying At High AOA Condition

\section{Independent Variable - Peripheral Cues}

To test for peripheral cue effects during approach/landing, surface and departure operations, two visibility levels were tested. The peripheral cues were either absent - simulating IMC of $200 \mathrm{ft}$ runway visual range (RVR) - or present - simulating Visual Meteorological Conditions (VMC) of 3 miles visibility.

\section{Evaluation Tasks}

\section{Approach}

The approach task mimicked an existing visual arrival procedure reflecting an efficient and preferred routing for air traffic control and noise abatement. This approach normally requires visual flight conditions for the crew to see-and-avoid terrain, traffic, and obstacles while navigating with respect to ground references. The approach was a curved, descending path around terrain and obstacles and, thus, tests the ability of the display concepts to support this type of equivalent visual operation. The weather consisted of altitude-based cross winds (wind direction and intensity was dependent on altitude), light turbulence, and varying visibility levels ( 3 miles or $200 \mathrm{ft} R V R$ ). The EP hand-flew the base and final legs of the visual arrival to RNO Runway 16R, using the HUD or XVS display concept with autothrottles engaged, holding 132 knots. The aircraft was configured for landing (landing gear down and flaps 30 degrees) prior to each run, and the aircraft was "cleared to land". The path converged into the instrument landing system approach course, nominally resulting in a stabilized approach no lower than 1,000 $\mathrm{ft} \mathrm{HAT}$. For the low AOA runs, the pilot was instructed to follow a pre-briefed taxi clearance requiring the aircraft to exit the runway on a high-speed turnoff onto Taxiway November, turn right on Taxiway Alpha, cross over Runway $7 / 25$, and then turn left on Taxiway Lima where the run ended. For the high AOA runs, the pilot was instructed to come to a full-stop on Runway 16R where the run ended.

\section{Departure}

EPs also performed departures flying the RNO "Mustang 7" Departure Procedure. They maintained the runway heading of 168 degrees until waypoint RIJTU (about $5 \mathrm{nmi}$ from the departure runway) and then turned left direct toward the Mustang VORTAC, where the run ended. The weather consisted of altitude-based cross winds (wind direction and intensity was dependent on altitude), light turbulence, and varying visibility levels ( 3 miles or $200 \mathrm{ft} R V R$ ). The EP hand-flew the departure with the HUD, XVS with HUD FOV or XVS with Full FOV display concept and was instructed to climb to $10,000 \mathrm{ft}$ mean sea level (MSL) and 250 knots.

There were up to 3 transport-sized aircraft in the runway environment, but they did not provide any conflicts for the ownship during approach, landing, taxi, or departure operations. There was no Air Traffic Control involvement in the tasks.

\section{Pilot Procedures}

Since only pilot-flying evaluations were being conducted, automatic aural altitude call-outs (e.g., $1000,500,100$ feet, etc.) were included in the simulation to "assist" in altitude awareness. Unlike current FAA regulations when landing with EV imagery on a HUD, for this experiment, the EP was not required to see using natural vision the required landing visual references (as per FAR $\$ 91.175$ ) by 100 $\mathrm{ft} \mathrm{HAT}$. The EP was instructed to continue to landing if the required landing visual references were seen in the imagery on the HUD or XVS and if the EP determined that a safe landing could be performed. Otherwise, a missed approach should be executed.

The EPs were instructed to fly the aircraft as if there were passengers aboard, fly the center of the approach path (within $\pm 1 / 2$ dot for desired performance and within \pm 1 dot for adequate performance), and land as close as possible to the centerline and aim point (1000 feet from the threshold). After landing, they were to capture the center line and then for the low 
AOA runs only, taxi at a speed with which they were comfortable using the pre-briefed taxi clearance. They were also instructed to initiate a go-around if the landing was not safe or if there were any safety concerns during the approach. EPs were instructed to stop the aircraft if they felt unsafe during surface operations.

Prior to run commencement, the EP was briefed on the type of run to be completed, the display concept to be evaluated, the visibility level, and the wind magnitude and direction.

\section{Experiment Matrix}

Nominally, ten training runs and twenty-seven experimental runs were completed by each EP. Of the 27 experimental runs, 5 non-normal runs were included to investigate the pilot's awareness and reaction to unexpected events and conditions (e.g., failures). The non-normal data are critical determinants in the underlying safety of all-weather operations. Due to paper page-limit constraints, these data are not reported herein.

For approach and landing runs, the experiment matrix (Table 1) consisted of a full-factorial combination of display type (HUD, XVS with HUD FOV, or XVS with Full FOV), AOA (low or high) and peripheral cues in side windows (absent or present). The MALSR ALS (Medium-Intensity Approach Lighting System with Runway Alignment Indicator) was held fixed for the approach and landing runs listed in Table 1.

Table 1. Approach and Departure Run Matrix

\begin{tabular}{|r|c|c|c|}
\hline & HUD & XVS with & XVS with \\
\hline \hline VMC Low AOA Approach & $\checkmark$ & Full FOV \\
\hline IMC Low AOA Approach & $\checkmark$ & $\checkmark$ & $\checkmark$ \\
\hline VMC High AOA Approach & $\checkmark$ & $\checkmark$ & $\checkmark$ \\
\hline IMC High AOA Approach & $\checkmark$ & $\checkmark$ & $\checkmark$ \\
\hline VMC Departure & $\checkmark$ & $\checkmark$ & $\checkmark$ \\
\hline IMC Departure & $\checkmark$ & $\checkmark$ & $\checkmark$ \\
\hline
\end{tabular}

Four additional runs were conducted to test for display type (HUD or XVS with Full FOV) and approach lighting system (VFR, ALSF-2 [Approach Lighting System with Sequenced Flashing Lights]) effects. The low AOA condition and the IMC visibility level were held fixed for these comparisons.
These four runs were compared to the 2 analogous MALSR ALS runs (HUD and XVS with Full FOV) from the Table 1 experimental matrix to test for ALS effects. Due to page limitations, the results for the ALS analyses are not reported herein.

For departure runs, the experiment matrix (Table 1) consisted of a full-factorial combination of display type and peripheral cues in the side windows for a total of 6 runs. The low AOA condition was held fixed for the departure runs.

Five non-normal runs were flown by each EP. The number of non-normal scenarios was designed to avoid expectancy on the part of the flight crew. The non-normal runs were two flight director failures (annunciated and unannunciated), two flare cue failures (annunciated and unannunciated), and one rejected takeoff. The EPs were trained to recognize the annunciated flight director failure, annunciated flare cue failure, and rejected takeoff. For the failures on approach, they were instructed to continue the landing if they felt it was safe to do so. For the rejected takeoff, they were instructed to stop the aircraft as quickly as possible on the runway. For the annunciated failure runs, the affected symbol (flight director or flare cue) was removed from the display and a failure flag was presented on the display. For the unannunciated failure runs, the affected symbol was frozen on the display and no failure flag was presented on the display. The rejected takeoff run was caused by a simulated right engine fire annunciated aurally. A between-subjects design was employed for the nonnormal approach and departure runs. As mentioned before, the results of the non-normal run analyses are not reported herein.

\section{Measures}

During each approach and landing run, path error, pilot control inputs, and touchdown performance (fore or aft of touchdown zone, and distance left or right of centerline) were measured for analysis. During taxi operations, centerline tracking and taxi speed were measured. For departure runs, centerline tracking, heading and climb rate maintenance, and altitude capture were measured.

After each run, pilots completed a run questionnaire consisting of the NASA Task Load Index (TLX) workload rating[10], Situation Awareness Rating Technique (SART)[10], and six Likert-type (5point) questions specific to different constructs of 
making a stabilized and safe approach to landing, taxiing (when appropriate), or departure.

After data collection was completed, pilots were administered two paired comparison tests: the Situation Awareness - Subjective Workload Dominance (SASWORD) [11] and Subjective Workload Dominance (SWORD) [10] techniques. The pilots also completed a post-test questionnaire to elicit comments on using the different display concepts, with and without peripheral cues, for conducting 1) low and high AOA approaches without a visual segment, 2) surface operations and 3) departures.

\section{Test Conduct}

The subjects were given a 1-hour briefing to explain the experiment purpose, HUD and XVS concepts, pilot procedures, and the evaluation tasks. After the briefing, a 1-hour training session in the IFD was conducted to familiarize the subjects with the aircraft handling qualities, display symbologies, pilot procedures, and controls. The annunciated flight director and flare cue failure runs and rejected landing runs were discussed and trained. The pilot's responsibility for maintaining safe operations at all times was stressed. Data collection lasted approximately 4.5 hours and was followed by debriefings which included the SWORD/SA-SWORD paired comparisons tests and a final questionnaire. The entire session including lunch and breaks lasted approximately 8 hours.

\section{Results}

For the objective performance measures and postrun questions, analysis of variance (ANOVA) were conducted for the factors of display type (HUD, XVS with HUD FOV, XVS with Full FOV), AOA (low, high) and peripheral cues (absent, present) for approach/landing operations. For surface and departure operations, only the low AOA condition was tested in this experiment so ANOVAs were conducted on the main factors of display type and peripheral cues. For the post-test paired comparisons, simple ANOVAs were conducted for display type. When necessary, Student-Newman-Keuls (SNK) post-hoc tests were performed with statistical significance assumed $a$ priori using $\alpha$ set at 0.05 .

\section{Approach Performance}

Approach performance was assessed using rms (root mean square) localizer deviation (in dots) and rms glide slope deviation (in dots). These parameters correspond intuitively to the establishment and maintenance of a stabilized approach to landing - an important safety measure.

The approach data were analyzed from $1000 \mathrm{ft}$ to $200 \mathrm{ft}$ HAT for the normal runs that ended in a landing. The beginning altitude value was chosen since the pilots were instructed to have the aircraft stabilized on the approach by $1000 \mathrm{ft} \mathrm{HAT} \mathrm{else,} \mathrm{they} \mathrm{should} \mathrm{perform}$ a go-around. The ending altitude value of $200 \mathrm{ft}$ was chosen as the lowest altitude of a typical instrument approach procedure.

\section{Localizer angular deviation}

The main factor of $\mathrm{AOA}(\mathrm{F}(1,22)=5.344, \mathrm{p}<0.05)$ and the interaction between AOA and peripheral cues $(F(1,22)=12.980, p<0.01)$ were significant for $\mathrm{rms}$ localizer deviation but none of the remaining main factors (display type, peripheral cues) or second-order interactions were significant for this measure. Pilots flew more precise lateral path in the low AOA (mean $=0.034$, standard deviation, $\sigma=0.028$ ) condition than in the high AOA (mean=0.041, $\sigma=0.040$ ) condition. For the high AOA condition, the change in rms localizer deviation from 0.034 dots when peripheral cues were present to 0.048 dots when they were absent in the side windows was more dramatic (difference of 0.014 ) than the change of 0.002 dots between the two peripheral cue conditions for the low AOA condition.

\section{Glide slope angular deviation}

Display type $(\mathrm{F}(2,44)=7.85, \mathrm{p}<0.01), \quad$ AOA $(\mathrm{F}(1,22)=9.091, \quad \mathrm{p}<0.01), \quad$ peripheral cues $(F(1,22)=22.032, \quad p<0.001), \quad$ and the interaction between display type and peripheral cues $(\mathrm{F}(2$, $44)=3.416, p<0.05$ ) were significant for this measure but the remaining second order interactions were not significant. Post-hoc tests revealed two overlapping subsets for glideslope tracking with display type: 1) XVS with Full FOV (mean $=0.141$ dots, $\sigma=0.096$ ) and XVS with HUD FOV (mean $=0.169, \sigma=0.114$ ) and 2) XVS with HUD FOV and HUD (mean $=0.184$, $\sigma=0.130$ ). Pilots had significantly better glide path performance with the XVS with Full FOV display than with the HUD display but neither of these concepts had appreciable differences with the XVS with HUD FOV 
display. Additionally, pilots flew more precise glide path: 1 ) in the low AOA condition (mean $=0.145$ dots, $\sigma=0.087)$ than in the high AOA condition (mean $=0.185$ dots and $\sigma=0.135$ ) and 2) when peripheral cues were present (mean $=0.138$ dots and $\sigma=0.088$ ) compared to when they were absent (mean $=0.192$ dots and $\sigma=0.132$ ). A reduction of 0.05 dots in rms glideslope deviation between the larger size XVS display (mean $=0.20$ ) and the smaller sized (HUD FOV) displays (mean $=0.015$ ) when no peripheral cues were present caused the interaction between display type and peripheral cues to be significant. The deviation was 0.13 dots for all 3 displays when there were peripheral cues in the side windows.

\section{Objective Approach Standards Analysis}

The Joint Aviation Authorities (JAA) Joint Aviation Requirement (JAR) All Weather Operations (AWO) performance-based approach standard for goaround rate (AWO-202) in low-visibility approaches with decision heights below $200 \mathrm{ft}$ and down to $100 \mathrm{ft}$ was also applied in the objective data analysis [12]. Specifically, the standards specify that no more than $5 \%$ of the approaches will have localizer deviations greater than $1 / 3$ dot or glideslope deviations greater than 1 dot between $300 \mathrm{ft}$ and $100 \mathrm{ft}$ HAT for certification acceptance. These low-visibility approach standards were not written specifically as quantitative performance standards for advanced vision systems (S/EV and XVS) operations, but are applied herein for comparative purposes.

The Continuous Method [12] technique was employed to calculate the probability of success, $P(\alpha)$, of meeting the AWO exceedance criteria (1/3 dot localizer, 1 dot glideslope) with required levels of confidence with the different display concepts flown. The probabilities of success for meeting the AWO localizer and glideslope criteria are shown, broken down by AOA, display type and visibility condition, in Table 2.

The data in Table 2 shows that localizer tracking was maintained, irrespective of the display being flown, approach angle-of-attack, or the absence or presence of peripheral cues in the side windows. For the low AOA flight condition, glideslope tracking was better under the following conditions: 1) when flying with the larger FOV display concepts compared to the smaller (HUD-size) FOV display concepts, 2) with the color XVS HUD FOV display concepts compared to the monochrome HUD display concepts, and 3) when peripheral cues were present for the display concept. However, for the high AOA flight condition, glideslope tracking was essentially equivalent for the XVS with Full FOV concept and HUD concept which is in contrast to the size and color effects found for the low AOA condition. Having peripheral cues in the side windows also improved glideslope tracking performance in the high AOA condition for all display types tested. The only display concept successfully meeting the JAR AWO-202 localizer and glideslope criterion (greater than $95 \%$ of the time) was the low AOA XVS with Full FOV display concept flown with peripheral cues present in the side windows. This color, uncollimated, display concept showed conformal imagery over a $34^{\circ}$ vertical FOV.

Table 2. Probabilities of Success in Meeting the AWO Localizer and Glideslope Criteria without a Visual Landing Segment

\begin{tabular}{|c|c|c|c|c|c|}
\hline \multirow[t]{2}{*}{ Display } & \multirow[t]{2}{*}{$\mathbf{W x}$} & \multicolumn{2}{|c|}{ Localizer $P(\alpha)$} & \multicolumn{2}{|c|}{ Glideslope $\mathbf{P}(\alpha)$} \\
\hline & & $\begin{array}{l}\text { Low } \\
\text { AOA }\end{array}$ & $\begin{array}{l}\text { High } \\
\text { AOA }\end{array}$ & $\begin{array}{l}\text { Low } \\
\text { AOA }\end{array}$ & $\begin{array}{l}\text { High } \\
\text { AOA }\end{array}$ \\
\hline HUD & VMC & 100 & 100 & 78.2 & 88.2 \\
\hline HUD & IMC & 100 & 100 & 67.4 & 74.8 \\
\hline $\begin{array}{l}\text { XVS HUD } \\
\text { FOV }\end{array}$ & VMC & 100 & 100 & 94.3 & 85.4 \\
\hline $\begin{array}{l}\text { XVS HUD } \\
\text { FOV }\end{array}$ & IMC & 100 & 99.9 & 80.6 & 54.9 \\
\hline $\begin{array}{l}\text { XVS Full } \\
\text { FOV }\end{array}$ & VMC & 100 & 100 & 96.0 & 87.8 \\
\hline $\begin{array}{l}\text { XVS Full } \\
\text { FOV }\end{array}$ & IMC & 100 & 100 & 92.6 & 84.2 \\
\hline
\end{tabular}

Note: Approaches using MALSR ALS.

The Continuous Method was applied to data from a prior experiment [13] for comparison. In this prior experiment, pilots evaluated the use of SV on a HUD during an instrument approach but they were required to transition to a natural vision segment. (Note that the symbology used during the SV-HUD experiment was identical to that used for the low $A O A$ condition in the current experiment, but the approach was flown at 138 knots instead of 132 knots.) The analysis from the previous SV HUD experiment revealed that $100 \%$ of the runs met the localizer criteria but only $60 \%$ of the runs met the glideslope criteria. The comparison of the 
two experiments (for the low AOA condition) shows that slight improvements in glideslope tracking may be realized with elimination of the visual segment $(60 \%$ vs. $67 \%$ ). The differences are not large, but the trends make intuitive sense. By allowing the pilot to concentrate on the task and display information, improved flight tracking performance results. The visual segment transition forces the pilots to simultaneously perform glideslope corrections and acquire the required landing visual references through normal vision.

\section{Objective Landing Standards Analysis}

Existing JAR AWO [12] performance-based landing standards (AWO 131) for longitudinal position and lateral position from centerline were applied in the objective landing data analysis. Specifically, the standards state that no longitudinal touchdown earlier than a point on the runway $200 \mathrm{ft}$ from the threshold or beyond $2700 \mathrm{ft}$ from the threshold and no lateral touchdown with the outboard landing gear more than $70 \mathrm{ft}$ from the runway centerline to a probability of $1 \mathrm{x}$ $10^{-6}$. These standards pertain to the general concept of low-visibility approach and landings, but were not written specifically for operations with advanced vision systems such as S/EV or XVS.

This experiment used an aim point located $1000 \mathrm{ft}$ from the runway threshold. For the simulated 757 aircraft, the outboard landing gear would be $70 \mathrm{ft}$ from the centerline when the fuselage (the recorded lateral landing position reported herein) is at $58 \mathrm{ft}$ lateral deviation from centerline, assuming no crab angle at touchdown.

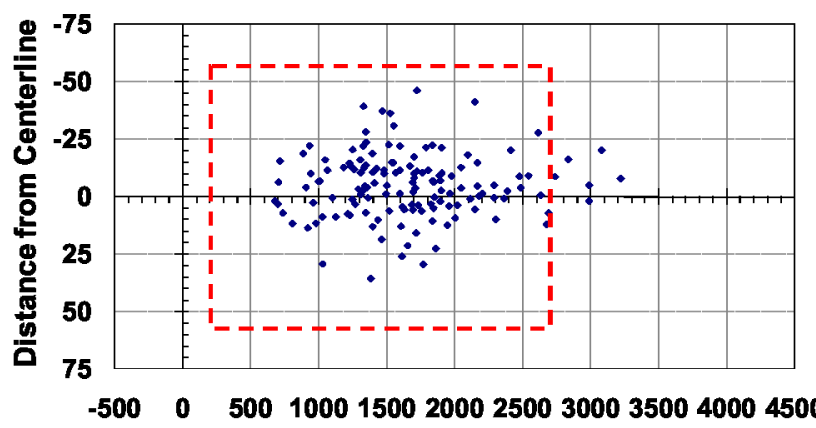

HUD

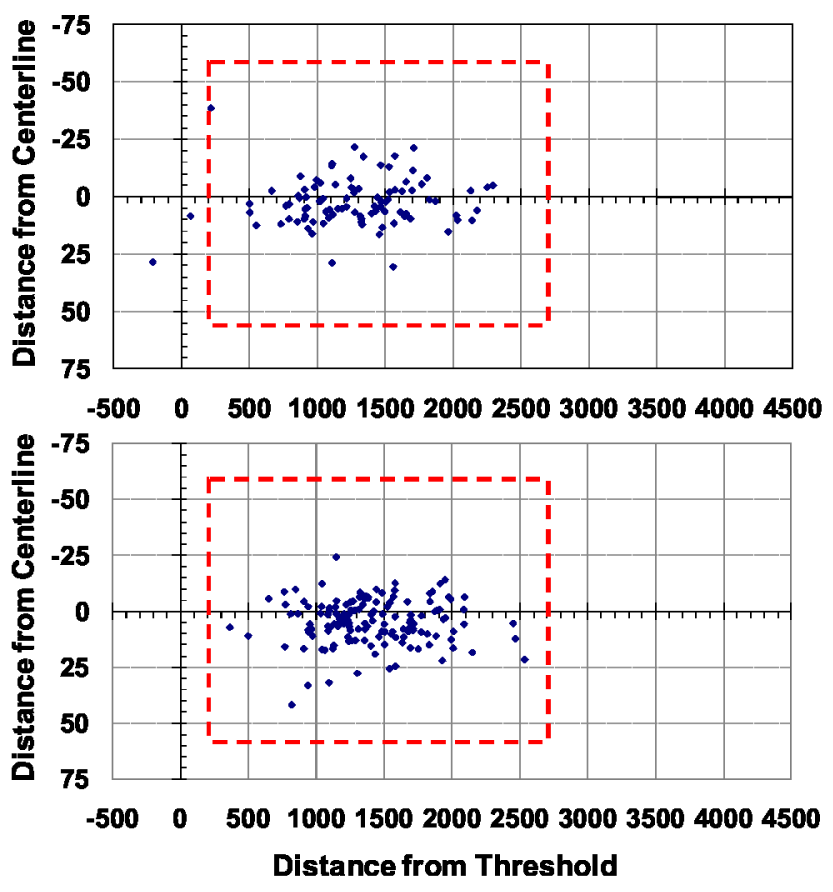

XVS HUD FOV

XVS Full FOV

Figure 6. Touchdown Data for Display Concepts 
In Figure 6, the touchdown data are shown, broken out by display concept but collapsed across AOA and peripheral cue influence. Included on this plot (in red, dashed rectangle) is the $+/-58 \mathrm{ft}$ lateral and $200 \mathrm{ft}$ to $2700 \mathrm{ft}$ longitudinal touchdown footprint defined in the JAR AWO landing criteria.

Visual inspection of the data shows that 1) all display concepts were within the lateral deviation from centerline touchdown footprint and 2) all the XVS with Full FOV display concept runs (i.e., both low AOA and high AOA runs) were within the JAR longitudinal position touchdown footprint. The XVS with HUD FOV concept had all but two runs (both using high AOA condition in IMC) within the touchdown box. However, these two runs were problematic as one was only $65 \mathrm{ft}$ past the threshold and the other actually landed $200 \mathrm{ft}$ short of the threshold (i.e., aircraft didn't land on the runway). All HUD runs were made on the runway but 6 runs (4 low AOA and 2 high AOA) were beyond the $2700 \mathrm{ft}$ from threshold criteria.

These data were analyzed against the $1 \times 10^{-6}$ probability requirements. The analyses show that the lateral landing position meets the $10^{-6}$ probability criteria (i.e., within $58 \mathrm{ft}$ of centerline) for: 1) the low AOA condition for all display types (XVS and HUD) when peripheral cues were present; 2) the low AOA condition for the XVS displays regardless of display size when peripheral cues were absent; 3) the high AOA condition with peripheral cues for the XVS with HUD FOV display; and 4) the high AOA condition without peripheral cues for the XVS Full FOV display. (Note: The high AOA condition with peripheral cues for the XVS Full FOV display almost met the required $1 \times 10^{-6}$ probability level with a value $5.8 \times 10^{-6}$.)

Satisfying the JAR AWO (low-visibility landing) longitudinal touchdown criteria to a $1 \times 10^{-6}$ probability was not met with any of the display concepts tested.

\section{Approach and Landing Performance Discussion}

Elimination of the visual segment of the approach had no adverse affects on localizer tracking as it was excellent from $1000 \mathrm{ft}$ to $200 \mathrm{ft} \mathrm{HAT}$ and well within JAR AWO approach criteria for decision heights below $200 \mathrm{ft}$ and down to $100 \mathrm{ft}$ regardless of display size (large FOV or HUD FOV), AOA (low or high), or whether the pilot had peripheral cues or not. However, glideslope tracking from the required stabilized approach altitude of $1000 \mathrm{ft}$ to $200 \mathrm{ft} \mathrm{HAT}$ appears to be affected by display size, AOA, and whether the pilot has peripheral cues in the side windows. Not surprisingly, glideslope tracking improvements were seen 1) with the larger FOV displays, 2) in the low AOA condition, and 3) when pilots had peripheral cues in the side windows. Glideslope tracking became less precise due to the 1) smaller sized displays, 2) symbology clustering in the high AOA condition, and 3 ) the lack of peripheral cues in the side windows. In fact, JAR AWO glideslope tracking criteria was only met by the low AOA condition in the XVS with Full FOV display concept with peripheral cues in the side windows. By comparison to another experiment - for the low AOA condition - elimination of the visual segment appears to have improved a pilot's glideslope tracking compared to previous experiments requiring a visual segment. This improvement can most likely be attributed to the fact that the pilots didn't have to simultaneously perform glideslope corrections and find required visual landing references in order to continue a landing.

The landing touchdown data shows that better performance was obtained with the XVS, using its full field-of-view. All touchdowns occurred within the AWO touchdown box. The HUD touchdown data showed 6 long landings and the XVS with a reduced (HUD-size) FOV had two short landings, including one unsafe landing. This unsafe landing was made with the XVS with HUD FOV concept in the high AOA condition and the pilot may have confused the displaced threshold for the aim point. In the high AOA condition, the flight path marker, guidance cue, and localizer deviation scale/indicator are clustered together at the bottom of the smaller FOV displays. It should be noted that the symbology was optimized for the low AOA condition and not the high AOA in this experiment. For actual supersonic operations, the symbology would be optimized for the flight display being used. Pilot commentary suggests that this symbology cluster increased workload and made it harder to discern the aim point within the imagery of the smaller sized displays. In total, 383 of the 384 landings were safely made to the runway with 376 of them within the JAR touchdown footprint. The lateral touchdown data shows that the JAR AWO criteria were met with $1 \times 10^{-6}$ probability for the XVS Full FOV display in all conditions but one. For the one condition, for the high AOA, with peripheral cues, the lateral landing criteria was nearly met. Lateral 
positioning for touchdown was not a problem across any of the experimental display concepts.

\section{Mental Workload}

Mental workload was assessed after each experimental run, using the NASA TLX, and post-test, using the SWORD technique.

NASA TLX is a multi-dimensional rating procedure that derives an overall workload score based on a weighted average of ratings on six subscales (mental demand, physical demand, temporal demand, performance, effort, and frustration level). The NASA TLX subscales went from 0 (Low) to 100 (High) for the workload ratings. TLX ratings were provided for every data run and configuration.

Pilots were administered the paired-comparison SWORD scale that enabled comparative ratings of mental workload. Mental workload was defined for the pilots as "the amount of cognitive resources available to perform a task and the difficulty of that task." The pair-comparison test was structured to compare the effects of angle-of-attack and XVS display type (i.e., Low AOA/XVS with HUD FOV, Low AOA/XVS with Full FOV, High AOA/XVS with HUD FOV, High AOA/XVS with Full FOV) flown by the EP. Note that this comparison does not include the collimation/color effects. Only the color, uncollimated XVS configurations were considered.

\section{NASA TLX}

Approach and Landing Operations: An ANOVA revealed significant differences for the main factors of display type $(\mathrm{F}(2,46)=11.07, \quad \mathrm{p}<0.001), \quad$ AOA $(F(1,23)=34.13, \quad p<0.001)$, and peripheral cues $(F(1,23)=16.32, \quad p<0.001)$ for the approach TLX ratings.

Post-hoc tests revealed that there were 2 unique subsets for display type with the XVS with Full FOV concept (mean=32) rated as having less workload then either the XVS with HUD FOV (mean=37) or the HUD (mean=37) concepts but with no appreciable differences in workload between the two smaller-sized display concepts. Workload was rated as being lower for the low AOA condition (mean=32) compared to the high AOA condition (mean $=39$ ) and when peripheral cues were available in the side window (mean=33) compared to when they were not (mean=38). There were no significant $(p>0.05)$ differences among any of the interactions for this measure.
Surface Operations: An ANOVA revealed that display $(F(2,44)=12.90, p<0.001)$ and peripheral cues $(F(1,22)=10.23, p<0.01)$ were significant for workload ratings. Post-hoc tests showed two unique subsets for workload ratings: 1) XVS with Full FOV concept (mean=23) having the lowest workload and 2) XVS with HUD FOV concept (mean=28) and HUD concept $(m e a n=32)$ with the highest workload during surface operations. Pilots rated their workload significantly lower when they had peripheral cues (mean=26) while taxiing compared to when they did not have them (mean=29).

Departure: An ANOVA revealed that the peripheral cues factor $(F(1,23)=5.04, p<0.05)$ was significant for pilot departure workload ratings, but display type (overall mean=29) was not significant $(p>0.05)$ for this measure. Pilots rated their workload lower when they had peripheral cues (mean $=28$ ) in the side windows during a departure compared to when they didn't have them (mean=30).

\section{SWORD}

The post-test paired-comparison SWORD data indicated that $A O A /$ display type $(F(3,92)=331.78$, $p<0.001$ ) was highly significant for the pilot ratings of mental workload. Post-hoc tests (SNK using $\alpha=0.05$ ) showed three unique subsets for the mental workload ratings with the $4 \mathrm{AOA} /$ display type combinations: 1) Low AOA/XVS with Full FOV (lowest workload); 2) High AOA/XVS with Full FOV and Low AOA/XVS with HUD FOV; and 3) High AOA/XVS with HUD FOV (highest workload).

\section{Workload Discussion}

The subjective post-run ratings indicated the workload was significantly reduced when using the larger displays (i.e., greater FOV) and when peripheral cues were available OTW for approach and surface operations. Workload was also rated lower for the low AOA condition compared to the high AOA condition during approach/landing runs. In consideration of the mean rating differences however, the workload changes created by these effects do not appear to be operationally significant. In all cases, the pilot comments and TLX numerical values indicated that the workload was very manageable when performing approach and landing using any of the evaluated concepts. This result is somewhat surprising for surface operations, since, in IMC conditions (200 RVR) and with the smaller FOV displays in particular, usable visual information is provided to the pilot only 
in very limited areas (the HUD and side windows). These surface operations results were likely influenced by the PI serving as a second crew-member, aiding the EP during right taxi turns. These results may have been dramatically different if it was a true single pilot operation. During departures, no workload differences were found for display size (i.e., FOV) but there were workload improvements when peripheral cues were added. Even though statistically significant workload differences were found, operationally these differences were not substantial - all were ranked at a moderately low workload level on the TLX scale.

The subjective post-test ratings revealed that display size was a larger influence in workload ratings than the AOA-induced differences. These results were supported by subjective commentary made during the post-test interviews.

\section{Situation Awareness}

Situation awareness was assessed after each experimental run, using the post-run SART, and posttest, using the SA-SWORD measures.

SART is a multi-dimensional rating technique using the constructs of: 1) demand on attentional resources; 2) supply of attentional resources; and 3) understanding. From these components, the SART rating is "understanding" reduced by the difference of "demand" minus "supply" (i.e., SART = \{(understanding) - (demand - supply)\}). SART ratings were provided for every data run and configuration.

Similar to the SWORD described above, the SASWORD is a paired-comparison technique that provides relative situation awareness ratings. For these comparisons, SA was defined as "the pilot's awareness and understanding of all factors that will contribute to the safe flying of their aircraft under normal and nonnormal conditions." The pair-comparison test was structured to compare the effects of angle-of-attack and XVS display type (i.e., Low AOA/XVS with HUD FOV, Low AOA/XVS with Full FOV, High AOA/XVS with HUD FOV, High AOA/XVS with Full FOV) flown by the EP. Note that this comparison does not include the collimation/color effects. Only the color, uncollimated XVS configurations were considered. The SA-SWORD measure differs from the post-run SART measure construct as it is a paircomparison test, it was administered post-test for all configurations, and the underlying definition and construct for the SA ratings are different.

\section{SART}

Approach and Landing Operations: An ANOVA revealed that the main factors of display type $(\mathrm{F}(2,46)=18.32, \quad \mathrm{p}<0.001), \quad$ AOA $\quad(\mathrm{F}(1,23)=48.28$, $\mathrm{p}<0.001)$, and peripheral cues $(\mathrm{F}(1,23)=21.16$, $p<0.001$ ) were highly significant for the approach SART ratings. Post-hoc tests revealed two unique subsets for display type with the XVS with Full FOV concept rated as providing more SA than either the XVS with HUD FOV or the HUD concepts, but with no appreciable differences in SA between the two smaller-sized display concepts. In addition, SA was rated as being higher 1) in the low AOA condition compared to the high AOA condition and 2) when peripheral cues were present in the side windows compared to when there were none. There were no significant $(p>0.05)$ differences among any of the interactions for this measure.

Surface Operations: An ANOVA revealed that display type $(F(2,44)=25.03, p<0.001)$ was highly significant for pilot taxi SART ratings, but peripheral cues was not significant ( $p>0.05)$ for this measure. Post-hoc tests showed three unique subsets for the SA ratings with the XVS with Full FOV concept providing the highest SA, followed by the XVS with HUD FOV concept, and then finally the HUD concept providing the least SA during surface operations.

Departure: An ANOVA revealed that the main factors, display type and peripheral cues, were not significant $(p>0.05)$ for pilot departure SART ratings.

\section{SA-SWORD}

The post-test SA-SWORD data indicated that AOA/display type $(F(3,92)=162.11, p<0.001)$ was highly significant for the pilot ratings of SA. Post-hoc tests (SNK using $\alpha=0.05$ ) showed four unique subsets for the SA ratings with the $4 \mathrm{AOA}$ /display type combinations: 1) Low AOA/XVS with Full FOV (highest SA); 2) High AOA/XVS with Full FOV; 3) Low AOA/XVS with HUD FOV; and 4) High AOA/XVS with HUD FOV (lowest SA).

\section{Situation Awareness Discussion}

The subjective post-run ratings indicated that SA was significantly enhanced when using the larger displays compared to the smaller ones for approach, landing and surface operations. In addition, SA ratings were greater when using the low AOA condition 
compared to the high AOA condition during approach and landing runs. SA improvements due to having peripheral cues in the side windows were only realized during approach and landing operations. This result for surface operations is somewhat counter-intuitive given the importance of full field-of-regard information for taxi path and traffic awareness on the airport but they, too, were probably influenced by the PI aiding the EP during surface operations.

Similar to the workload results, the subjective post-test ratings where display type was held fixed (i.e., only comparing XVS/AOA display combinations) revealed display size was a stronger influence on SA than AOA. These results were also supported by pilot comments during the post-test interviews.

\section{Discussion}

An experiment was conducted to investigate the use of S/EV and XVS technologies as enabling technologies for future all-weather operations. The experimental objectives were to determine the feasibility of these advanced vision technologies to provide for all weather (visibility) landing capability without the need (or ability) for a visual approach segment and to determine the interaction of these vision technologies (S/EV or XVS) with peripheral vision cues for terminal area and surface operations. Objective results indicate that elimination of the visual segment of the approach had no adverse affects on localizer tracking or lateral landing position with respect to the centerline as both were excellent regardless of the display type (XVS, HUD) and angleof-attack (low, high) condition being evaluated or whether or not there were peripheral cues in the side windows. However, glideslope tracking from the required stabilized approach altitude of $1000 \mathrm{ft}$ to 200 $\mathrm{ft}$ height above touchdown (HAT) appears to be affected by display size, angle-of-attack (AOA), and whether the pilot has peripheral cues in the side windows. Not surprisingly, glideslope tracking improvements were seen: 1) with the larger FOV displays, 2) in the low AOA condition, and 3) when pilots had peripheral cues in the side windows. In fact, JAR AWO glideslope tracking criteria was only met by the low AOA condition in the XVS with Full FOV display concept with peripheral cues in the side windows. For the low AOA condition, elimination of the visual segment appears to have improved a pilot's glideslope tracking compared to previous experiments requiring a visual segment. This improvement can most likely be attributed to the fact that the pilots didn't have to simultaneously perform glideslope corrections and find required visual landing references in order to continue a landing.

The landing touchdown performance data requires more thorough examination and exploration. In the low AOA condition, the XVS display concepts, regardless if there were peripheral cues in the side windows or not, met existing performance-based lateral touchdown position landing criteria. The HUD concepts in the low AOA condition, only met these criteria when peripheral cues were present. The XVS display concepts, with full FOV, all fell within the current standard for landing touchdowns, but the dispersions were too large to meet a $10^{-6}$ probability condition. More importantly, touchdowns happened outside this touchdown footprint for the HUD and XVS with a HUD FOV display conditions. Additional analysis and experimentation is necessary to evaluate if this result was the consequence of the experiment protocol (i.e., pilots were not instructed that they must land within the touchdown box) or if the display characteristics are good enough to met the standard.

Not surprising, subjective results indicate that a larger display size provides significantly more situation awareness during approach and surface operations than smaller size displays. Workload reductions during approach and landing operations were also found with the larger size displays compared to the smaller ones, but not during departure operations. SA and workload improvements were also seen during approach and landing operations when using the low AOA condition compared to the high AOA condition. Having peripheral cues in the side windows significantly reduced pilot workload during all operational phases tested, but these workload differences appear operationally inconsequential. Similar results for peripheral cues were found for SA. These results are somewhat surprising given the importance of full fieldof-regard information for the surface operations task (e.g., awareness and maintenance of the taxi path; separation from other traffic; and ground hazards). Further investigation of these results is necessary. Paired comparison tests where display type (XVS/AOA display concepts) was held fixed revealed that display size was a larger influence in workload ratings than AOA. These results were supported by subjective commentary made during the post-test interviews. 
The data showed little evidence of a display collimation effect (i.e., when comparing XVS-HUD vs. HUD). In this experiment, this effect was confounded by the display color differences, but there was little subjective commentary noting an influence of display collimation. The data suggests that the effect of collimation was a second-order effect compared to the color differences between the HUD and XVS display. (It was impractical in this test to completely divorce the collimation and display color variables, so the effects are confounded.) If this result is validated, a less-complex non-collimated display could be suitable for an XVS-type system; however, these results need to be validated by motion-based simulation and flight testing where vestibular and peripheral vision effects are properly stimulated. Approach, landing and surface operations with these conformal head-up display concepts using advanced vision technologies should be explored to evaluate the effects of motion and peripheral cuing on collimation, groundspeed awareness, and ground rush/sink rate perception.

\section{References}

[1] Kramer, L. J. and Norman, R. M. (2000). High-speed research surveillance symbology assessment experiment (Tech. Memo. No. NASA TM2000-210107). Washington, DC: NASA.

[2] Kramer, L. J, Parrish, R. V, Williams, S. P., and Lavell, J. S. (1999). Effects of inboard horizontal field of view display limitations on pilot path control during total in-flight simulator (TIFS) flight test (Tech. Rep. No. NASA TP-1999-209542). Washington, DC: NASA.

[3] Joint Planning and Development Office. (2008). Next generation air transportation system integrated plan: a functional outline. Washington, DC: Author.

[4] National Aeronautics and Space Administration. (n.d.). Aviation safety program/integrated intelligent flight deck technical plan summary. Retrieved October 3, 2007, from $\mathrm{http}: / / \mathrm{www}$.aeronautics.nasa.gov/nra_pdf/iifd_tech_pla n_cl.pdf

[5] Bailey, R. E., Kramer, L. J., \& Prinzel, L. J., III. (2006). Crew and display concepts evaluation for synthetic/enhanced vision systems. Proceedings of
SPIE, Enhanced and Synthetic Vision Conference 2006, 6226, 62260G.1-62260G.18.

[6] Bailey, R. E., Kramer, L. J., \& Prinzel, L., III. (2007). Fusion of synthetic and enhanced vision for allweather commercial aviation operations (Tech. Rep. No. NATO RTO-HFM-141). In NATO Human Factors and Medicine Symposium on Human Factors and Medical Aspects of Day/Night All Weather Operations: Current Issues and Future Challenges (pp. 11-1-11-18). Neuilly-sur-Seine, France: RTO.

[7] Kramer, L. J., Prinzel, L. J., III, Arthur, J. J., III, \& Bailey, R. E. (2005). Advanced pathway guidance evaluations on a synthetic vision head-up display (Tech. Rep. No. NASA TP-2005213782).Washington, DC: NASA.

[8] Merrick, V.K. and Jeske, J.A. (1995). Flightpath synthesis and HUD scaling for V/STOL terminal area operations. (Tech. Memo. No. NASA TM-1995-110348). Washington, DC:NASA.

[9] Federal Aviation Administration (1993). Standard operating procedures for flight deck crewmembers (Advisory Circular 25-773-1). Washington, DC: Author.

[10] AIAA (1993). Guide to Human Performance Measurement. Washington D.C.: American Institute of Aeronautics and Astronautics.

[11] Vidulich, M.A. and Hughes, E.R. (1991). Testing a subjective metric of situation awareness. In Proceedings of the Human Factors \& Ergonomics Society, 35th Annual Meeting (pp. 1307-1311). Santa Monica, CA: Human Factors Society.

[12] Joint Aviation Authorities. (2007). Joint Aviation Requirements, All-Weather Operations (Amendment 4). Englewood, CO: Global Engineering Documents.

[13] Kramer, Lynda J.; Williams, Steven P.; \& Bailey, Randall E. (2008). Simulation evaluation of synthetic vision as an enabling technology for equivalent visual operations. In Proceedings of SPIE Enhanced and Synthetic Vision Conference 2008, $6957,69570 \mathrm{~K}-1-69570 \mathrm{~K}-15$.

\section{8th Digital Avionics Systems Conference}

October 25-29, 2009 